\title{
HYDROALCOHOLIC EXTRACT OF SWERTIA CHIRATA AND SWERTIA CORDATA ATTENUATES HYPOXIA-MEDIATED MEMORY DYSFUNCTION BY IMPROVING NEURONAL SURVIVAL IN WISTAR RATS
}

\author{
KRITIKA KAUSHAL ${ }^{1}$, HARVINDER SINGH ${ }^{2}$, ANIL KANT ${ }^{1 *}$ \\ ${ }^{1}$ Department of Biotechnology and Bioinformatics, Jaypee University of Information Technology, Waknaghat, Solan, Himachal Pradesh,
} India. ${ }^{2}$ Leaders Institute, 76 Park Road, Woolloongabba, Brisbane, Australia. Email: anil.thakur@juit.ac.in

Received: 16 August 2018, Revised and Accepted: 03 October 2018

\begin{abstract}
Objective: Swertia chirata and Swertia cordata have been used in traditional and folk medicines to treat several mental disorders. However, the mechanistic and experimental justification to its traditional use is lacking. The present study was aimed to investigate the neuromodulatory potential of $S$. chirata and $S$. cordata during hypoxia-induced neuronal damage in Wistar rats and to determine the underlying mechanism.

Methods: Animals were divided into six groups $(\mathrm{n}=5)$. Hypoxia was inflicted by subjecting animals to the atmosphere having $10 \% \mathrm{O}_{2}$ for 3 days. Animals were administered $100 \mathrm{mg} / \mathrm{kg}$ hydroalcoholic extract of $S$. chirata and S. cordata orally once daily for 7 days, after which motor coordination (Rotarod test) and memory functions (active avoidance test and passive avoidance test) were evaluated. Animals were sacrificed and biochemical investigations for oxidative stress and histopathology were performed.

Results: Subjecting animals to hypoxia resulted in marked memory dysfunction, and extract treatments improved memory functions in active avoidance and passive avoidance task. Hypoxiainduced the marked oxidative stress as indicated by the significantly elevated reactive oxygen species and lipid peroxidation and depleted catalase and glutathione levels in the hippocampus. S. chirata and S. cordata treatment alleviated oxidative stress in the hippocampus region of the brain. Brain histopathology confirmed that hypoxia resulted in significant neuronal damage and extract treatment efficiently rescued neurons from hypoxic damage. Overall, $S$. chirata extract treatment was observed to have better neuromodulatory effect than $S$. cordata during hypoxia.
\end{abstract}

Conclusion: Hypoxia induced memory dysfunction by inflicting neuronal damage and oxidative stress in the hippocampus region of the brain. The hydroalcoholic extract of $S$. chirata and $S$. cordata improved memory functions in hypoxic animals by alleviating hippocampal oxidative stress and by improving neuronal morphology and survival.

Keywords: Swertia chirata, Swertia cordata, Oxidative stress, Neurotoxicity, Hippocampus, Learning and memory.

(c) 2019 The Authors. Published by Innovare Academic Sciences Pvt Ltd. This is an open access article under the CC BY license (http://creativecommons. org/licenses/by/4. 0/) DOI: http://dx.doi.org/10.22159/ajpcr.2019.v12i2.29131

\section{INTRODUCTION}

Hypoxia is associated with the development of hippocampal neurodegeneration-mediated memory dysfunction and other behavioral deficit $[1,2]$. Hypoxia results in reduced oxygen supply to the cells, resulting in generation of excessive free radicals, such as superoxide, hydroxyl, and peroxynitrite radicals, in the mitochondrial electron transport chain [3]. These free radicals are neutralized by the antioxidants and radical scavenging enzymes present in our body; however, neutralizing capacity of these mechanisms is limited and therefore gets saturated, leading to the development of oxidative stress. The excessive free radicals circulate in the body and interact with biomolecules such as proteins, DNA, RNA, and lipids compromising their normal physiology and functioning, leading to the development of complications such as memory dysfunction $[4,5]$. The brain is one of the major consumers of oxygen which makes it highly vulnerable to the oxidative stress-mediated damage, especially hippocampus region which is involved in continuous neurogenesis and memory functions [6-8]. Hippocampal neurodegeneration results in impaired memory functions and other behavioral dysfunctions such as depression, anxiety, and Alzheimer's disorder. [6,8,9]. Antioxidant treatment is reported to improve behavioral functions by alleviating oxidative stress and improving neuronal survival and functions $[5,8,10]$.

Despite well-known association of hypoxia and oxidative stress with the development of neurological complications, there is not even single drug available till date for the management of oxidative stress. Therefore, some alternative and safer treatment strategy is urgently needed which can alleviate oxidative stress during hypoxia and other conditions such as diabetes and chronic stress and rescue neurons from degenerations and associated complications. Swertia chirata and S. cordata are well-known medicinal plants in Ayurveda, which are known for their beneficial effects during hepatitis, liver disorders, inflammation, chronic fever, malaria, anemia, GIT disorders, hypertension, mental disorders, and diabetes [11,12]. Till date, numerous scientific studies have experimentally justified the traditional use of these plants for hepatic and other disorders [13]; however, their use in the mental disorders remains unexplored so far, besides, the mechanisms through which the beneficial effects on the brain are achieved remain unknown. Therefore, the present study was designed to get and insight to the neuromodulatory effect of $S$. chirata and $S$. cordata during hypoxia in Wistar rats and explore the oxidative stress as a potential mechanism.

\section{METHODS}

Material

Analytical grade chemicals and reagents were used in this study. All the chemicals and reagents were procured from Sigma, Hi-Media, and Loba Chemie, unless otherwise specified on the first mention. Kits for the measurement of oxidative stress parameters were procured from Roche Diagnostics (Roche Diagnostics K.K., Tokyo, Japan). UV-spectrophotometric recordings were taken on NanoDrop Spectrophotometer (ND-2000C, Thermo Scientific). 
Plant collection and extract preparation

S. chirata and S. cordata whole plants were collected between July and September from the high altitude regions of Himachal Pradesh and Uttarakhand (India). Both plants were identified by local herbal medical practitioners and authenticated by Prof. N. S. Chauhan, Dr. Y. S. Parmar University of Horticulture and Forestry, Solan, Himachal Pradesh, India. Plants were washed to remove dirt and shade dried at room temperature until constant weight was achieved and coarsely powdered. Plants were subjected to hydroalcoholic extraction (80:20 - methanol:water) inside Soxhlet extractor at $25^{\circ} \mathrm{C}$ for $72 \mathrm{~h}$. Extracts of both plants were filtered while hot, concentrated using rotary evaporator, and lyophilized. The extractive yield was calculated and the extract was stored at $-20^{\circ} \mathrm{C}$ in a refrigerator until used for further analysis.

\section{Animals}

The entire study was performed on male Wistar rats. Experimental procedures were approved by the Institutional Animal Ethics Committee (IAEC) (DIHAR/IAEC/36/2015). Experiments were performed strictly as per the guidelines of CPCSEA, Government of India. Wistar rats (150-200 g; 8-10 months of age) were housed in the DIHAR animal house inside polypropylene cages ( 2 animals per cage) under standard conditions of temperature $\left(23 \pm 2^{\circ} \mathrm{C}\right), 12 \mathrm{~h}$ day/light cycle, and relative humidity $(65 \pm 5 \%)$. Animals had free access to food and water.

\section{Animal groupings}

Animals were divided into the following groups.

Group 1- Control (CTRL) (received $0.3 \%$ carboxymethyl cellulose as a vehicle orally)

Group 2- CTRL $+100 \mathrm{mg} / \mathrm{kg}$ S. chirata extract suspended in a vehicle (SCA 100)

Group 3- CTRL + $100 \mathrm{mg} / \mathrm{kg} \mathrm{S.} \mathrm{cordataextract} \mathrm{suspended} \mathrm{in} \mathrm{a} \mathrm{vehicle}$ (SCO 100)

Group 4- Hypoxia (HYP) (rats exposed to 10\% oxygen for 3 days and received vehicle orally)

Group 5- HYP + $100 \mathrm{mg} / \mathrm{kg}$ S. chirata extract suspended in a vehicle (HYP-SCA 100)

Group 6- HYP + $100 \mathrm{mg} / \mathrm{kg}$ S. cordata extract suspended in a vehicle (HYP-SCO 100)

Each group had 5 animals, and all the treatments were given orally once daily between 6 and 7 pm for 7 days as per the groupings. Behavioral studies were performed $24 \mathrm{~h}$ after the last dose to avoid interference from the possible acute drug effect.

\section{Induction of hypoxia}

Animals were subjected to hypoxia inside a custom-made hypoxia chamber. This chamber was linked to oxygen $\left(\mathrm{O}_{2}\right)$ and nitrogen $\left(\mathrm{N}_{2}\right)$ cylinders. The concentration of $\mathrm{O}_{2}$ inside the chamber or the concentration of $\mathrm{O}_{2}$ to which animals were exposed was regulated by $\mathrm{N}_{2}$. In this study, hypoxia was inflicted by exposing rats to $10 \% \mathrm{O}_{2}$ for 3 days.

\section{Rotarod test}

Effect of hypoxia and extract treatments on muscle coordination was evaluated through Rotarod test according to the method described by Mehta et al. [10]. Rotarod test is one of the most extensively used procedures to evaluate muscle coordination and strength in rodents. Animals were brought to the experimental room 30 min before the experimentation to get them acclimatized to the laboratory conditions. Each animal was placed on the rotating bar of the Rotarod ( $30 \mathrm{rpm})$, and the time taken to fall from the bar was recorded. Each animal was given three trials at an interval of 5 min with a maximum cutoff time of $180 \mathrm{~s}$. The best time recorded was taken as the final reading to evaluate muscle coordination. Entire apparatus was wiped with $70 \%$ ethanol between every experimental session to avoid any sort of olfactory cues.

\section{Memory function tests}

Passive avoidance test

Effect of extract treatments on the hypoxia-mediated memory dysfunction was evaluated through passive avoidance paradigm as per the method described previously [5], with few modifications as per the requirements of our study. The apparatus consisted of two adjacent wooden chambers. One of the chambers was painted white and was brightly illuminated by $100 \mathrm{~W}$ bulb placed 3 feet above the chamber. Other chamber was painted black and was covered to provide a dark environment to the animals. Both chambers were interconnected through a small opening $(5 \mathrm{~cm} \times 5 \mathrm{~cm})$, which provided free access to the animals to enter any chamber. Animals were brought to experimental room illuminated by low-intensity light $30 \mathrm{~min}$ before the start of learning trial. Each animal was placed in the light chamber and time taken to enter the dark chamber was recorded, with a maximum cutoff of $180 \mathrm{~s}$. As soon as the animal entered the dark chamber, an inescapable foot shock was delivered through grid floor. Animals were immediately removed and returned to their home cages. Memory retention was evaluated $24 \mathrm{~h}$ after the learning trials. Animals were again placed in the light chamber and the time taken to enter the dark chamber was recorded, with a maximum cutoff of $180 \mathrm{~s}$. However, this time no foot shock was delivered. Entire apparatus was wiped with $70 \%$ ethanol between every experimental session to avoid any sort of olfactory cues.

\section{Active avoidance test}

Effect of hypoxia and extract treatments on memory acquisition and retention was evaluated through active avoidance paradigm as per the method described by Moscarello and LeDoux [14], with few modifications as per the requirements of our study. The apparatus consisted of two adjacent wooden chambers painted white. Both chambers were having grid floor capable of delivering foot shock and were interconnected through a small opening $(5 \times 5 \mathrm{~cm})$, which provided free access to the animals to enter any chamber. In one of the randomly selected chambers, animals were given scrambled electric foot shock while the other served as a non-shock chamber. Animals were brought to the experimental room illuminated by low-intensity light, $30 \mathrm{~min}$ before the start of learning experiment. Each animal was placed in the random chamber and was given conditioned stimulus through a buzzer for $2 \mathrm{~s}$ followed by a buzzer and foot shock of $0.1 \mathrm{~mA}, 40 \mathrm{~V}$ for $4 \mathrm{~s}$. To avoid the foot shock, if rat moved to a shock-free chamber, the avoidance was considered positive. If rat failed to move, it was gently guided to the shock-free chamber for learning. This process was repeated thrice, and then, animals were returned to their home cages. After $24 \mathrm{~h}$, animals were again brought to the experimentation room for memory retention trials. Each animal was placed into the random chamber of the apparatus and was given conditioned stimulus, and the time taken to reach safe chamber was recorded as the avoidance behavior, with the maximum cutoff of $180 \mathrm{~s}$. No foot shock was given during the retention trials. Entire apparatus was wiped with $70 \%$ ethanol between every experimental session to avoid any sort of olfactory cues.

\section{Animal sacrifice and sample collection}

For biochemical evaluation, animals were sacrificed by cervical dislocation, the brain was dissected out, and hippocampus was isolated, weighed, and homogenized in HEPES buffers and stored at $4^{\circ} \mathrm{C}$ until used. All the samples were used within $6 \mathrm{~h}$ for the biochemical evaluations. For histopathological examinations, animals were anesthetized with $90 \mathrm{mg} / \mathrm{kg}$ ketamine $+5 \mathrm{mg} / \mathrm{kg}$ xylazine followed by fixation with $2 \%$ formaldehyde $+2 \%$ glutaraldehyde solution using double circulation technique. Tissues were collected and stored in fixing solution at room temperature until used further $[5,8]$.

\section{Mitochondrial reactive oxygen species (ROS) generation}

Effect of hypoxia and extract treatment on mitochondrial ROS generation was evaluated as per the method described by Wasilewski and Wojtczak [15], with required modifications. Briefly, amount of protein in the hippocampal homogenate was quantified using Bradford method. $15 \mu \mathrm{g}$ of protein was incubated with the reaction buffer ( $5 \mathrm{mM}$ pyruvate, $2.5 \mathrm{mM}$ malate, and $10 \mathrm{M}$ of dichlorodihydrofluorescein diacetate) for $25 \mathrm{~min}$ at $37^{\circ} \mathrm{C}$. Level of fluorescence generated as a result of interaction of dichlorodihydrofluorescein diacetate with ROS was quantified using a CaryEclipse fluorimeter (Varian, Palo Alto, USA) at excitation wavelength of $485 \mathrm{~nm}$ and emission wavelength of $582 \mathrm{~nm}$. Results were normalized to protein content. 
Levels of lipid peroxidation

Effect of extract treatment on hypoxia-mediated lipid peroxidation was assayed according to the method of Mehta et al. [5], with slight modifications. Briefly, hippocampal homogenate equivalent to $15 \mu \mathrm{g}$ protein was diluted with equal volume of $0.1 \mathrm{M}$ Tris-HCl buffer having $\mathrm{pH}$ 7.4. The resulting mixture was incubated for $2 \mathrm{~h}$ at $37^{\circ} \mathrm{C}$ with constant mixing inside an incubator. $1 \mathrm{ml}$ of trichloroacetic acid $(10 \% \mathrm{w} / \mathrm{v})$ was added to the reaction mixture, mixed, and centrifuged at 10,000 rpm for $7 \mathrm{~min}$. Further, $1.5 \mathrm{ml}$ of thiobarbituric acid $(0.67 \% \mathrm{w} / \mathrm{v})$ was added to each tubes, followed by incubation over a water bath maintained at $95^{\circ} \mathrm{C}$ for $10 \mathrm{~min}$. Reaction tubes were cooled to room temperature and centrifuged at 10,000 rpm for $15 \mathrm{~min}$, supernatant was collected, and the absorbance was recorded at $532 \mathrm{~nm}$ using UV spectrophotometer. The results were expressed as nmol TBARS/mg protein.

\section{Catalase activity}

Effect of hypoxia and extract treatments on the hippocampal catalase activity was assayed as per the method described by Mehta et al. [5]. Catalase activity was recorded spectrophotometrically at $240 \mathrm{~nm}$, and the results were expressed as moles of hydrogen peroxide $\left(\mathrm{H}_{2} \mathrm{O}_{2}\right)$ consumed per minute. Hippocampal homogenate equivalent of $15 \mu \mathrm{g}$ protein was diluted to $0.5 \mathrm{ml}$, and $32 \mu \mathrm{l}$ of $30 \% \mathrm{H}_{2} \mathrm{O}_{2}$ was added to it. Absorbance was immediately recorded and the readings were taken every minute for $4 \mathrm{~min}$. The level of catalase in the hippocampal homogenate was determined in terms of the amount of $\mathrm{H}_{2} \mathrm{O}_{2}$ consumed during the 4-min reaction interval.

\section{Glutathione (GSH) levels}

Effect of hypoxia and extract treatments on the hippocampal GSH peroxidase activity was determined using the method of Necheles et al. [16], with few modifications. Reaction mixture was prepared by mixing $0.4 \mathrm{ml}$ phosphate buffer $(0.35 \mathrm{M} ; \mathrm{pH} 7.4), 0.1 \mathrm{ml}$ sodium azide $(10 \mathrm{mM}), 0.2 \mu$ freshly prepared GSH $(8 \mathrm{mM}), 0.5 \mathrm{ml}$ hippocampal homogenate equivalent to $15 \mu \mathrm{g}$ protein, $0.1 \mathrm{ml} \mathrm{H}_{2} \mathrm{O}_{2}(2.5 \mathrm{mM})$, and $1.1 \mathrm{ml}$ double-distilled water in respective tubes. These tubes were then incubated for $5 \mathrm{~min}$ at $37^{\circ} \mathrm{C}$ followed by addition of $0.5 \mathrm{ml}$ trichloroacetic acid $(10 \%)$. Reaction mixture was centrifuged at $3500 \mathrm{rpm}$ for $15 \mathrm{~min}$ at room temperature, supernatant was isolated, and $0.5 \mathrm{ml}$ supernatant was diluted with $3 \mathrm{ml}$ of disodium hydrogen phosphate $(0.3 \mathrm{M}) .1 \mathrm{ml}$ freshly prepared DTNB (in phosphate buffer $\mathrm{pH}$ 7.4) was added to the reaction mixture, and the reaction mixture was allowed to stand for $5 \mathrm{~min}$ at room temperature. Absorbance was recorded at $412 \mathrm{~nm}$ using a UV spectrophotometer and the results were expressed as mg of GSH consumed $/ \mathrm{min} / \mathrm{mg}$ protein.

\section{Histopathological examination}

Effect of extract treatments on the hypoxia-mediated neuronal damage was evaluated through histopathological examination, as per the method described previously [5,8]. Briefly, the brain of the animals was processed as described above. Fixed brain samples were embedded into the paraffin wax, and thick section of $5 \mu \mathrm{m}$ was prepared by microtome. Sections were fixed to glass slides, which were then stored at room temperature until used. Neuronal integrity and damage were detected by hematoxylin-eosin staining method. Images of the sections were captured at $\times 100$ magnification, and the effect of hypoxia and extract treatments on neuronal morphology was determined.

\section{Statistical analysis}

Statistical significance of the results was analyzed using GraphPad Prism 6. All values are expressed as mean \pm standard error of the mean. Statistical significance was determined by one-way analysis of variance followed by Dunnett's post hoc test at ${ }^{*} \mathrm{p}<0.05,{ }^{* *} \mathrm{p}<0.01$, and ${ }^{* * *} \mathrm{p}<0.001$ versus control and ${ }^{\#} p<0.05,{ }^{\# \#} p<0.01$ and ${ }^{\# \# \#} p<0.001$ versus HYP.

\section{RESULTS}

\section{Extractive yield and phytochemical evaluation}

The crude extractive yield of the hydroalcoholic Soxhlet extraction of S. chirata and S. cordata was observed to be $22.37 \%$ and $26.32 \%$, respectively. To get an idea of the phytochemicals in the extracts, we performed qualitative phytochemical screening which revealed the presence of alkaloids, flavonoids, terpenoids, glycosides, steroids, tannins, and saponins as the major ingredients. Moreover, volatile oil, fixed oil, proteins, and acidic compounds were not observed to be present in the plant extracts in significant amounts. We, further, observed that both the extracts were having high amount of flavonoids and phenolic compounds which might be responsible for its antioxidant potential.

\section{Behavioral investigation \\ Rotarod test}

The effect of hypoxia and extract treatments on the muscle coordination was evaluated through Rotarod test, and the results are depicted in Fig. 1a. Subjecting animals to hypoxia resulted in a non-significant impairment in muscle coordination as the time taken to fall from the rotating bar was not significantly different than control animals. Likewise, we did not observed any significant difference in the muscle coordination of control and hypoxic animals which were treated with plant extracts, when compared to CTRL or HYP. These results suggest that short-term hypoxia does not impair muscle coordination and animals had normal muscular activity.

\section{Passive avoidance test}

Effect of $S$. chirata and $S$. cordata extract treatment on the hypoxiamediated memory dysfunction was evaluated through passive avoidance step-through paradigm, and the results are depicted in Fig. 1b. Subjecting animals to hypoxia resulted in marked memory impairments. Our results demonstrated significantly $(p<0.001)$ lower latency to enter the dark chamber in HYP animals during retention trials, thereby suggesting that animals were not able to remember the foot shock they received in the dark chamber during learning trials. Extract treatment significantly improved memory performance in hypoxic animals. Animals were able to remember the foot shock and were resistant to enter the dark chamber. Animals were preferred to stay in light chamber and the transfer latencies were significantly $(p<0.01)$ higher than HYP. Further, control animals treated with plant extracts were also able to retain foot shock memory and transfer latencies did not showed any deviations from CTRL.

\section{Active avoidance}

Effect of $S$. chirata and $S$. cordata extract treatment on the hypoxiamediated learning and memory dysfunction was evaluated through active avoidance paradigm, and the results are depicted in Fig. 1c. Hypoxia significantly impaired learning and memory in animals. Hypoxic animals were not able to learn the association between auditory cue and foot sock. These animals demonstrated significantly $(\mathrm{p}<0.01)$ higher transfer latency to the safer region of the apparatus, suggesting impaired learning and memory. Extract treatment rescued animals from hypoxia-mediated memory dysfunction. These animals efficiently remembered the association between audio cue and foot shock. The transfer latencies of these animals were significantly $(\mathrm{p}<0.01)$ lower than the HYP animals. Moreover, control animals treated with plant extracts also demonstrated good learning and memory functions, and transfer latencies of these animals did not showed any deviations from CTRL animals.

\section{Biochemical investigation \\ Mitochondrial ROS generation}

The results of the mitochondrial ROS generation and the effect of S. chirata and S. cordata treatment are depicted in Fig. 2a. Hypoxiainduced significant oxidative stress in the hippocampus and the levels of hippocampal ROS were observed to be significantly $(\mathrm{p}<0.001)$ higher than the CTRL animals. The levels of hippocampal ROS in control animals treated with plant extract did not show any significant variations from the control animals, suggesting that extract treatment is not contributing directly to enhance ROS production in the hippocampus. Further, treating hypoxic rats with $100 \mathrm{mg} / \mathrm{kg}$ dose of $S$. chirata and $S$. cordata extract resulted in 


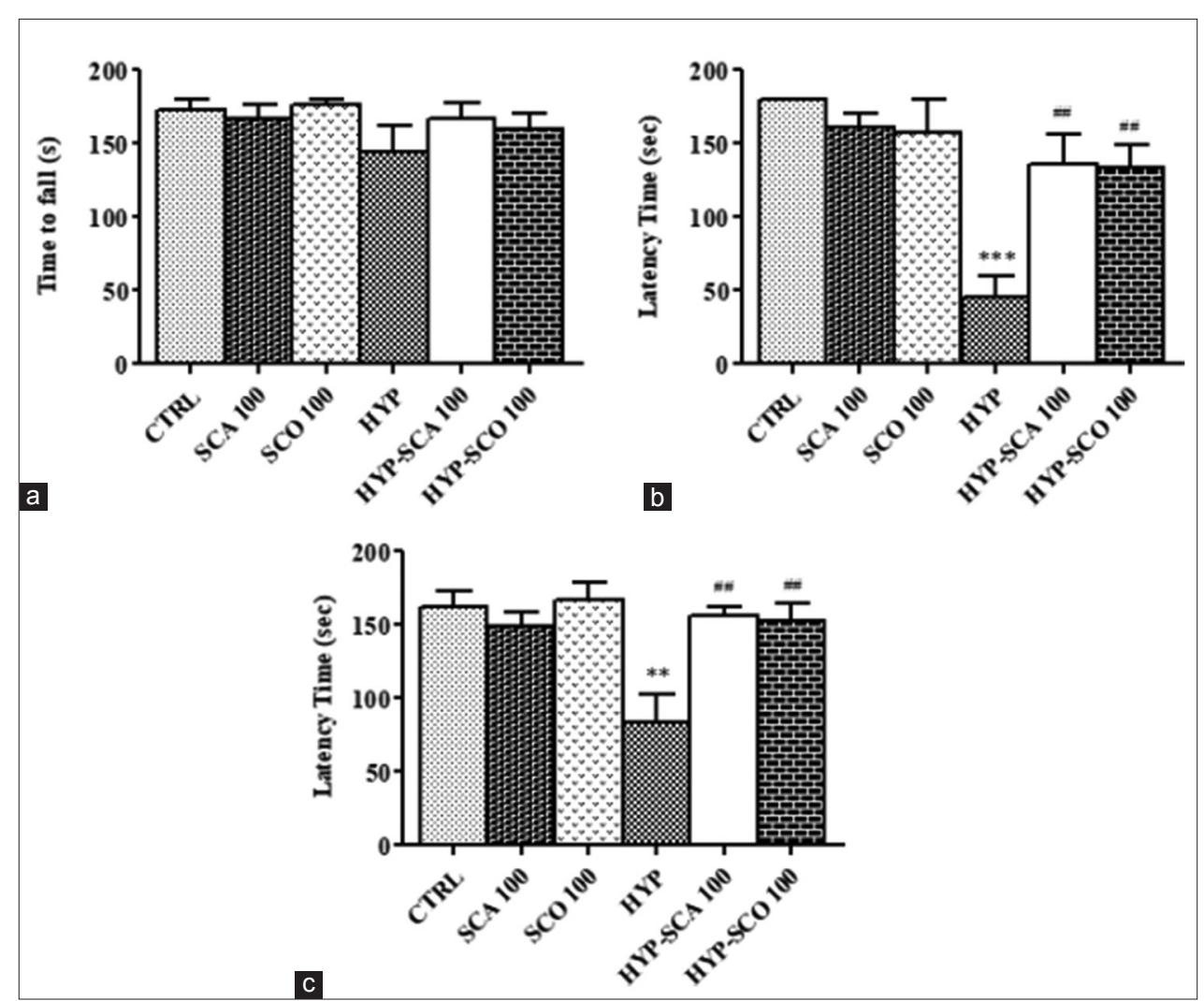

Fig. 1: Effect of Swertia chirata and Swertia cordata treatment on hypoxia-mediated behavioral alterations (a - Rotarod test; b - passive avoidance test; $c$ - active avoidance test). Values are represented as mean \pm standard error of the mean. CTRL: Control; SCA 100: Control $+100 \mathrm{mg} / \mathrm{kg}$ S. chirata extract; SCO 100: Control+100 mg/kg S. cordata extract; HYP: Hypoxia; HYP-SCA 100: HYP+100 mg/kg S. chirata extract; HYP-SCO 100: HYP $+100 \mathrm{mg} / \mathrm{kg}$ S. cordata extract. ${ }^{*} \mathrm{p}<0.05 ;{ }^{* *} \mathrm{p}<0.01 ;{ }^{* * *} \mathrm{p}<0.001$ versus CTRL group. ${ }^{\#} \mathrm{p}<0.05$; ${ }^{\# \#} \mathrm{p}<0.01$; $\# \#$ p $<0.001$ versus HYP group.

a significant $(\mathrm{p}<0.05)$ reduction in mitochondrial ROS generation when compared to HYP. Our results suggest that both the extracts are having comparable antioxidant potential, which may be attributed to high levels of flavonoid and phenolic compounds present in these plants.

\section{Lipid peroxidation}

Lipid peroxidation is a measure of damage to the cellular membrane. The biochemical levels of malondialdehyde (MDA) are estimated as a marker of lipid peroxidation in the biological samples such as brain, especially during the animal experimentation. The results of hypoxia-mediated lipid peroxidation and the effects of extract treatments are depicted in Fig. 2b. Our results suggest that hypoxia induced the marked lipid peroxidation in the hippocampus, as the levels of MDA were observed to be significantly $(\mathrm{p}<0.001)$ higher in HYP animals when compared to CTRL. Treating HYP animals with $S$. chirata and $S$. cordata extract reduced the lipid peroxidation levels, and the levels of MDA were observed to be significantly $(p<0.001)$ lower than HYP. Inhibition of lipid peroxidation was observed to be more pronounced in S. chirata-treated animals than animals treated with $S$. cordata extract. Moreover, control animals treated with these extracts did not show any significant difference when compared to CRTL, eliminating any possibility of the acute effect of plant extracts.

\section{Catalase activity}

Catalase is one of the most potent antioxidant enzymes present in our entire body, including the brain. It is actively involved in the detoxification of ROS generated during the functioning of electron transport chain. The results of the effect of hypoxia and extract treatments in hippocampal catalase levels are depicted in Fig. 2c. Subjecting animals to hypoxia resulted in the depletion of hippocampal catalase levels. Catalase levels were observed to be significantly $(\mathrm{p}<0.001)$ lower in HYP animals, when compared to CTRL animals. Treating hypoxic animals with plant extracts improved hippocampal catalase levels and our results demonstrated a significant $(p<0.01)$ increase in catalase levels when compared to HYP. Normal animals treated with plant extract did not demonstrate any significant deviation in catalase levels, when compared to CTRL, eliminating any possibility of direct effect of plant extracts. Our results suggest that $S$. chirata and S. cordata extracts are having a good antioxidant potential which can be partially attributed to its potential to either upregulate catalase activity or prevent its depletion during oxidative stress.

\section{GSH level}

The effect of hypoxia and extract treatment on hippocampal GSH levels was evaluated and the results are depicted in Fig. 2d. Subjecting animals to hypoxia resulted in a significant $(p<0.01)$ depletion or lowering of GSH levels in the hippocampus region of the brain. Our results demonstrated a comparable and marked antioxidant potential of extract treatments. Treating hypoxic animals with $S$. chirata and $S$. cordata extracts significantly $(\mathrm{p}<0.05)$ improved hippocampal GSH levels when compared to HYP. Normal animals treated with extract did not show any significant difference in hippocampal GSH levels when compared to CTRL, thereby suggesting that observed effects of treatments during hypoxic conditions are the result of rescuing hippocampus form hypoxia-mediated damage.

\section{Histopathological examination}

Hematoxylin-eosin staining is widely used method to determine the neuronal damage and morphology. We used this technique to demonstrate the effect of hypoxia on the neuronal morphology and evaluated the neuroprotective effect of $S$. chirata and $S$. cordata extract treatments. Results are depicted in Fig. 3. Analysis of the stained sections demonstrated the normal neuronal morphology in samples belonging to CTRL animals. HYP induced a marked neuronal damage and neurons appeared to be degenerated or damaged in significant number. Extract 


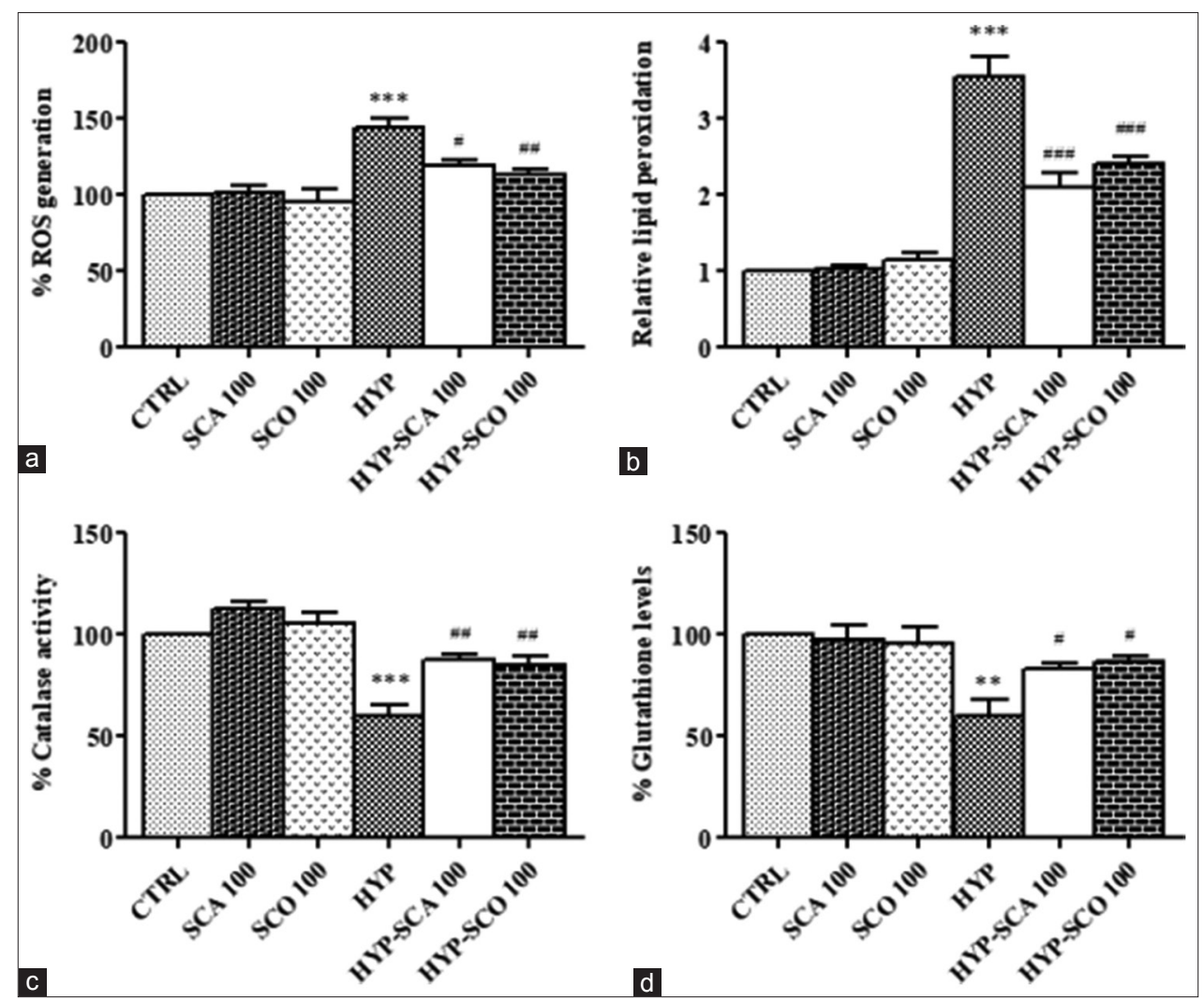

Fig. 2: Effect of Swertia chirata and Swertia cordata treatment on hypoxia induced oxidative stress (a - \% ROS generation; b - lipid peroxidation; $\mathrm{c}$ - \% catalase activity; $\mathrm{d}$ - \% glutathione levels). Values are represented mean \pm standard error of the mean. CTRL: Control; SCA 100: control + 100 mg/kg S. chirata extract; SCO 100: Control + 100 mg/kg S. cordata extract; HYP: Hypoxia; HYP-SCA 100: HYP $+100 \mathrm{mg} / \mathrm{kg}$ S. chirata extract; HYP-SCO 100: HYP $+100 \mathrm{mg} / \mathrm{kg}$ S. cordata extract. ${ }^{*} \mathrm{p}<0.05 ; * * \mathrm{p}<0.01 ;{ }^{* * *} \mathrm{p}<0.001$ versus CTRL group. ${ }^{\#} \mathbf{p}<0.05 ;{ }^{\# \#} \mathbf{p}<0.01 ;{ }^{\# \# \#} \mathbf{p}<0.001$ versus HYP group.

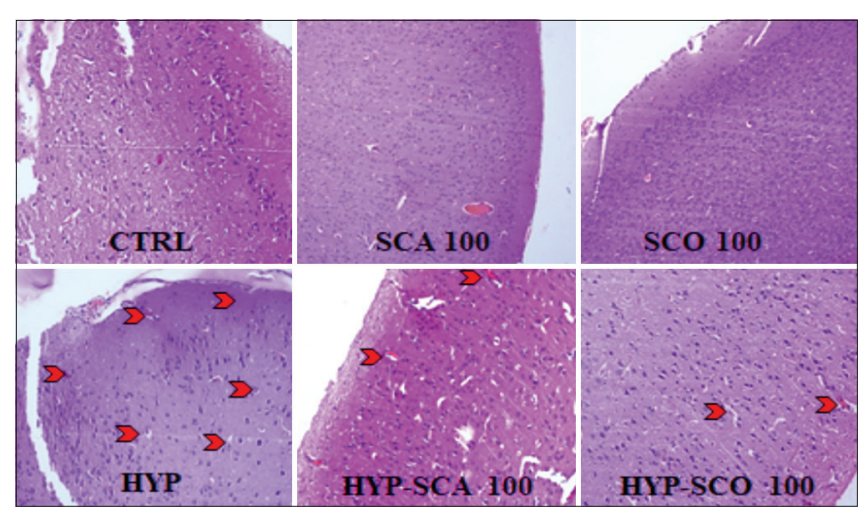

Fig. 3: Effect of Swertia chirata and Swertia cordata treatment on hypoxia-induced neuronal damage. Histopathological examination of brain sections using hematoxylin-eosin staining. Red arrow indicates the damage regions on the histopathological sections

treatments rescued neurons from hypoxia-mediated damage and neurons of these animals were having normal morphology resembling CTRL animals with lesser signs of damage and degeneration. These results concluded that $S$. chirata and $S$. cordata extract can protect neurons from hypoxia-mediated damage, which might have resulted in improved memory functions in these animals.

\section{DISCUSSION}

The present investigation demonstrated the improvement in memory functions, oxidative stress, and neurodegeneration by the hydroalcoholic extract of $S$. chirata and $S$. cordata treatment against hypoxia in Wistar rats. Hypoxia results in the generation of excessive free radicals, which surpasses the neutralizing capacity of the antioxidants present in our body, such as catalase, GSH, and superoxide dismutase. [4,17]. These free radicals then circulate in the body and damage the functioning of vital biomolecules, resulting in the development of neurological complications such as depression [18], cognitive dysfunction [19], Alzheimer's disorder [20], dementia [20], hepatotoxicity [17], cellular damage [21], necrosis [21], and apoptosis [22]. Moreover, these free radicals interact with the membrane phospholipids and induce lipid peroxidation, which further result in the cellular damage and degeneration $[4,5,17]$. Previous reports suggest that the hypoxia markedly impaired learning and memory functions in rodents [1,2]. Our results are in line with these reports. Herein, we demonstrated marked impairment in learning and memory in hypoxic Wistar rats during active avoidance and passive avoidance paradigm. Moreover, we observed high levels of ROS and lipid peroxidation with depleted catalase and GSH in the hippocampus of these animals, which is primarily associated with the memory and learning functioning in rodents. Many studies associate the development of behavior dysfunction with the enhanced oxidative stress [23-25], and alleviation of oxidative stress is demonstrated to result in improved behavior in animals [5,26-28]. We further demonstrated that treating hypoxic animals with $S$. chirata and $S$. cordata extracts reduced ROS generation and lipid peroxidation in the hippocampus and enhanced antioxidant levels. We further reported the presence of high levels of phenolic compounds and flavonoids in the hydroalcoholic extracts of these plants. Phenolic and flavonoids are potent antioxidants, and literature reports the neuromodulatory effect of herbal preparations which are rich in these antioxidants [29-31]. In our study, extract treatments significantly improved learning and memory, which may be the result of alleviation of the hippocampal oxidative stress. We did not observe any significant difference in the muscle 
coordination of various treatment groups, and thereby, any possibility of increased transfer latency in memory test as a result of muscular insufficiency is eliminated. Further, histopathological examination of the rat brain revealed that hypoxia induced a marked degeneration in the brain, which may probably be due to the enhanced oxidative stress and may be a crucial contributing factor in memory dysfunction. Previous reports suggest that neurodegeneration and neuronal damage are associated with impaired memory function in rodents $[5,10]$, besides indicating enhanced oxidative stress as an important contributing factor for neurodegeneration $[5,10]$. Neuroprotective potential of herbal interventions has been reported to be associated with the improved behavior functions [5,10,29-33]. Our results are in line with these findings, and we have demonstrated that treating hypoxic animals with plant extract improved neuronal morphology and rescued them from degenerative changes. These results suggest that the hydroalcoholic extract of $S$. chirata and $S$. cordata efficiently improved hypoxiamediated memory dysfunction which can be attributed to their potential to alleviate hypoxia-induced oxidative stress in the brain.

\section{CONCLUSION}

Hypoxia is associated with the development of memory dysfunction, enhanced oxidative stress, and neuronal damage in Wistar rats. The hydroalcoholic extract of $S$. chirata and S. cordata improved memory functions and demonstrated comparable neuroprotective potential which was associated with the attenuation of hypoxia-mediated oxidative stress. Our finding provides an experimental justification for the traditional use of these plants during brain disorders. Moreover, herbal preparations are safe to use and may find a clinical application for the management of neurological complications associated with oxidative stress. These findings are preliminary and need to be evaluated further.

\section{CONFLICTS OF INTEREST}

Authors declare no conflicts of interest.

\section{AUTHORS' CONTRIBUTIONS}

KK designed and performed experiments. She was involved in manuscript editing and finalization as well. HS and AK designed entire study project and contributed in experiment finalization and implementation, manuscript editing, and finalization.

\section{ACKNOWLEDGMENT}

Authors would like to acknowledge the Department of Biotechnology, Bioinformatics, and Pharmacy, JUIT, for providing all the resources to conduct this research. We are thankful to Dr. Sunil Kumar Hota and Surya of Defence Institute of High Altitude Research (DIHAR), DRDO,for their help in conducting animal experiments in their laboratory. We are also thankful to Prof. N. S. Chauhan of Dr. Y. S. Parmar University of Horticulture and Forestry, Solan, Himachal Pradesh for identification and authentication of plant species.

\section{REFERENCES}

1. Kossatz E, Silva-Peña D, Suárez J, de Fonseca FR, Maldonado R, Robledo P, et al. Octadecylpropyl sulfamide reduces neurodegeneration and restores the memory deficits induced by hypoxia-ischemia in mice. Front Pharmacol 2018;9:376.

2. Dong Y, Li Y, Feng D, Wang J, Wen H, Liu D, et al. Protective effect of HIF-1 $\alpha$ against hippocampal apoptosis and cognitive dysfunction in an experimental rat model of subarachnoid hemorrhage. Brain Res 2013;1517:114-21

3. Chhunchha B, Fatma N, Kubo E, Rai P, Singh SP, Singh DP, et al. Curcumin abates hypoxia-induced oxidative stress based-ER stressmediated cell death in mouse hippocampal cells (HT22) by controlling prdx6 and NF-kB regulation. Am J Physiol Cell Physiol 2013; 304:C636-55.

4. Mehta V, Malairaman U. Flavonoids: Prospective strategy for the management of diabetes and its associated complications. In: Handbook of Research on Advancing Health Education through Technology. Hershey, PA: IGI Global; 2016. p. 286-328.
5. Mehta V, Parashar A, Udayabanu M. Quercetin prevents chronic unpredictable stress induced behavioral dysfunction in mice by alleviating hippocampal oxidative and inflammatory stress. Physiol Behav 2017;171:69-78

6. Huang WJ, Zhang X, Chen WW. Role of oxidative stress in alzheimer's disease. Biomed Rep 2016;4:519-22.

7. Patel M. Targeting oxidative stress in central nervous system disorders. Trends Pharmacol Sci 2016;37:768-78.

8. Mehta V, Parashar A, Sharma A, Singh TR, Udayabanu M. Quercetin ameliorates chronic unpredicted stress-mediated memory dysfunction in male swiss albino mice by attenuating insulin resistance and elevating hippocampal GLUT4 levels independent of insulin receptor expression. Horm Behav 2017;89:13-22.

9. Bartsch T, Wulff P. The hippocampus in aging and disease: From plasticity to vulnerability. Neuroscience 2015;309:1-6.

10. Mehta V, Singh TR, Udayabanu M. Quercetin ameliorates chronic unpredicted stress-induced behavioral dysfunction in male swiss albino mice by modulating hippocampal insulin signaling pathway. Physiol Behav 2017;182:10-6

11. Kumar V, Van Staden J. A review of Swertia chirayita (Gentianaceae) as a traditional medicinal plant. Front Pharmacol 2015;6:308.

12. Roy P, Abdulsalam FI, Pandey DK, Bhattacharjee A, Eruvaram NR, Malik T, et al. Evaluation of antioxidant, antibacterial, and antidiabetic potential of two traditional medicinal plants of India: Swertia cordata and Swertia chirayita. Pharm Res 2015;7:S57-62.

13. Khan AU, Rahim A, Iqbal Z, Gilani AH. Insights into mechanisms underlying the gut and airways modulatory effects of Swertia chirata. J Nat Med 2012;66:140-8.

14. Moscarello JM, LeDoux JE. Active avoidance learning requires prefrontal suppression of amygdala-mediated defensive reactions. J Neurosci 2013;33:3815-23.

15. Wasilewski M, Wojtczak L. Effects of N-acylethanolamines on the respiratory chain and production of reactive oxygen species in heart mitochondria. FEBS Lett 2005;579:4724-8.

16. Necheles TF, Maldonado N, Barquet-Chediak A, Allen DM. Homozygous erythrocyte glutathione-peroxidase deficiency: Clinical and biochemical studies. Blood 1969;33:164-9.

17. Li S, Tan HY, Wang N, Zhang ZJ, Lao L, Wong CW, et al. The role of oxidative stress and antioxidants in liver diseases. Int J Mol Sci 2015; 16:26087-124.

18. Anderson G, Maes M. Oxidative/nitrosative stress and immunoinflammatory pathways in depression: Treatment implications. Curr Pharm Des 2014;20:3812-47.

19. Gan X, Wu L, Huang S, Zhong C, Shi H, Li G, et al. Oxidative stressmediated activation of extracellular signal-regulated kinase contributes to mild cognitive impairment-related mitochondrial dysfunction. Free Radic Biol Med 2014;75:230-40.

20. Tramutola A, Lanzillotta C, Perluigi M, Butterfield DA. Oxidative stress, protein modification and alzheimer disease. Brain Res Bull 2017; 133:88-96.

21. Choi K, Kim J, Kim GW, Choi C. Oxidative stress-induced necrotic cell death via mitochondira-dependent burst of reactive oxygen species. Curr Neurovasc Res 2009;6:213-22.

22. Mukherjee D, GhoshAK, BasuA, Datta S, Pattari SK, Bandyopadhyay A, et al. Beneficial role of melatonin in the complete recovery from isoproterenolinduced cardiac injury in rats. Int J Pharm Pharm Sci 2013; 5:561-9.

23. Lin MT, Beal MF. Mitochondrial dysfunction and oxidative stress in neurodegenerative diseases. Nature 2006;443:787-95.

24. Navarro A, Sánchez Del Pino MJ, Gómez C, Peralta JL, Boveris A. Behavioral dysfunction, brain oxidative stress, and impaired mitochondrial electron transfer in aging mice. Am J Physiol Regul Integr Comp Physiol 2002;282:R985-92.

25. Niizuma K, Endo H, Chan PH. Oxidative stress and mitochondrial dysfunction as determinants of ischemic neuronal death and survival. J Neurochem 2009;109 Suppl 1:133-8.

26. Farr SA, Poon HF, Dogrukol-Ak D, Drake J, Banks WA, Eyerman E, et al. The antioxidants alpha-lipoic acid and $\mathrm{N}$-acetylcysteine reverse memory impairment and brain oxidative stress in aged SAMP8 mice. J Neurochem 2003;84:1173-83.

27. Joseph JA, Shukitt-Hale B, Denisova NA, Bielinski D, Martin A, McEwen JJ, et al. Reversals of age-related declines in neuronal signal transduction, cognitive, and motor behavioral deficits with blueberry, spinach, or strawberry dietary supplementation. J Neurosci 1999; 19:8114-21.

28. Navarro A, Gomez C, López-Cepero JM, Boveris A. Beneficial effects of moderate exercise on mice aging: Survival, behavior, oxidative 
stress, and mitochondrial electron transfer. Am J Physiol Regul Integr Comp Physiol 2004;286:R505-11.

29. Kennedy DO, Wightman EL. Herbal extracts and phytochemicals: Plant secondary metabolites and the enhancement of human brain function. Adv Nutr 2011;2:32-50.

30. Krikorian R, Shidler MD, Nash TA, Kalt W, Vinqvist-Tymchuk MR, Shukitt-Hale B, et al. Blueberry supplementation improves memory in older adults. J Agric Food Chem 2010;58:3996-4000.

31. LeeYK, YukDY,LeeJW, LeeSY,HaTY,OhKW, etal. (-)-epigallocatechin- 3-gallate prevents lipopolysaccharide-induced elevation of beta-amyloid generation and memory deficiency. Brain Res 2009;1250:164-74.

32. Babu SM, Swain S, Renuka K. Neuroprotective activity of fractional flower extracts of Mirabilis jalapa against aluminium hydrochloride induced neurotoxicity in male Wister rats. Int J Pharm Pharm Sci 2017; 9:216-21.

33. Kassab RB, Bauomy AA. The neuroprotective efficency of the aqueous extract of clove (Syzygium aromaticum) in aluniniuminduced neurotoxicity. Int J Pharm Pharm Sci 2014;6:503-8. 